\title{
Using Logical Decision Trees for Clustering
}

\author{
Luc De Raedt and Hendrik Blockeel \\ Department of Computer Science, Katholieke Universiteit Leuven, \\ Celestijnenlaan 200A, B-3001 Heverlee, Belgium \\ email: \{Luc.DeRaedt,Hendrik.Blockeel $\} @$ cs.kuleuven.ac.be
}

\begin{abstract}
A novel first order clustering system, called $\mathrm{C} 0.5$, is presented. It inherits its logical decision tree formalism from the TILDE system, but instead of using class information to guide the search, it employs the principles of instance based learning in order to perform clustering. Various experiments are discussed, which show the promise of the approach.
\end{abstract}

\section{Introduction}

A decision tree is usually seen as representing a theory for classification of examples. If the examples are positive and negative examples for one specific concept, then the tree defines these two concepts. One could also say, if there are $k$ classes, that the tree defines $k$ concepts.

Another viewpoint is taken in Langley's Elements of Machine Learning [11]. Langley sees decision tree induction as a special case of the induction of concept hierarchies. A concept is associated with each node of the tree, and as such the tree represents a kind of taxonomy, a hierarchy of many concepts. This is very similar to what many clustering algorithms do (e.g. COBWEB, [9]).

Concept hierarchies can be induced in a supervised or unsupervised manner. Decision trees are an example of the former, while typical clustering algorithms are unsupervised.

The ILP system TILDE [2] induces first order logical decision trees from classified examples. In this paper, we show how to adapt the TILDE system to perform clustering, resulting in the C 0.5 system. To realize this, principles from instance based learning are employed. More specifically, we assume that a distance measure is given that computes the distance between two examples. Furthermore, in order to compute the distance between two clusters (i.e. sets of examples), we employ a function that computes a prototype of a set examples. A prototype can be regarded as an example. The distance between two clusters is then defined as the distance between the prototypes of the two clusters. Now, the distance measure employed determines whether the learning process is supervised or unsupervised. If the distance measure employs class information, learning is supervised, if no class information is employed, learning is unsupervised.

All logical aspects of TILDE are inherited by $\mathrm{C} 0.5$. This includes the logical representation of binary decision trees and the learning from interpretations setting (in which examples are logical interpretations). 


\section{The problem}

We use the learning from interpretations setting. In this setting, each example is a Prolog program encoding the specific properties of the example. See $[5,3]$ for more details on learning from interpretations.

For instance, examples for the well-known mutagenesis problem [14] can be described by interpretations. Here, an interpretation is simply an enumeration of all the facts we know about one single molecule: its class, lumo and logp values, the atoms and bonds occurring in it, certain high-level structures, ... We can represent it e.g. as follows: $\{\operatorname{logmutag}(-0.7), \operatorname{lumo}(-3.025), \operatorname{logp}(2.29)$, atom $\left(d 189 \_1, c, 22,-0.11\right)$, bond $\left.\left(d 189 \_2, d 189 \_3,7\right), \ldots\right\}$.

A logical decision tree is a binary decision tree in which each node contains a conjunction of literals. This conjunction may share variables with nodes above it in the tree. The test that is to be performed at the node consists of its conjunction, together with the conjunctions on the path from the root of the tree to this node that share variables with this node or with other nodes sharing variables with it. This test has two possible outcomes (it may fail or succeed), upon which the splitting of a cluster of examples into two subclusters is based.

The format of logical decision trees makes them perfectly fit as a representation for a cluster hierarchy. Each node in the tree corresponds to a cluster of examples, and the hierarchical structure of the tree shows how clusters are split into subclusters. The test in a node can be seen as a discriminant description of the two clusters in which the current cluster of examples is divided. One cluster is described by saying that the test succeeds, the other by saying that it fails.

This view is in correspondence with Langley's viewpoint that a test in a node is not just a decision criterion, but also a description of the subclusters formed in this node. In [2], we have shown how a logical decision tree can be transformed into an equivalent logic program. This procedure can be used to obtain descriptions of the clusters.

The purpose of conceptual clustering is to obtain clusters such that intracluster distance (i.e. the distance between examples belonging to the same cluster) is as small as possible and the inter-cluster distance (i.e. the distance between examples belonging to different clusters) is as large as possible.

In this paper, we assume that a distance measure $d$ that computes the distance $d\left(e_{1}, e_{2}\right)$ between examples $e_{1}$ and $e_{2}$ is given. Furthermore, there is also a need for measuring the distance between different clusters (i.e. between sets of examples). Therefore we will assume as well the existence of a prototype function $p$ that computes the prototype $p(E)$ of a set of examples $E$. The distance between two clusters $C_{1}$ and $C_{2}$ is then defined as the distance $d\left(p\left(C_{1}\right), p\left(C_{2}\right)\right)$ between the prototypes of the clusters. This shows that the prototype should be considered as (possibly) partial example descriptions.

For instance, on the mutagenesis problem, the distance could be the Euclidean distance $d_{1}$ between the activities of the two compounds, or the Euclidean distance $d_{2}$ between the points in the three-dimensional space corresponding to the lumo, logp and activity values of the two compounds, or it could be the distance $d_{3}$ as measured by a first order distance measure. 
Given the distance at the level of the examples, the principles of instance based learning can be used as to compute the prototypes. E.g. on the mutagenesis problem, $d_{1}$ would result in a prototype function $p_{1}$ that would simply compute the average activity of the compounds in the cluster, $d_{2}$ would result in a prototype function $p_{2}$ that would compute the average instance along the lumo, $\log p$ and activity values of the compounds in the cluster, whereas $d_{3}$ could result in function $p_{3}$ that would compute the (possibly reduced) least general generalisation ${ }^{1}$ of the compounds in the cluster.

Notice that although we employ - in this paper - only propositional distance measures, we obtain first order descriptions of the clusters through the representation of logical decision trees. Hence, one could say that we realize 0.5 th order Clustering, which explains the name of our system C 0.5.

By now we are able to formally specify the clustering problem:

\section{Given}

- a set of examples $E$ (each example is a definite clause theory),

- a background theory $B$ in the form of a definite clause theory,

- a distance measure $d$ that computes the distance between two examples or prototypes,

- a prototype function $p$ that computes the prototype of a set of examples,

Find: clusters in the form of a logical decision tree.

Notice that in this problem-setting, the interpretation $i$ corresponding to an example $e$ is the least Herbrand model of $B \wedge e$. So, conceptually, the clustering process learns from interpretations. This is similar to the Claudien [4], ICL [6] and TILDE [2] systems.

\section{C 0.5 : Clustering of order 0.5}

C 0.5 employs the basic TDIDT framework as it is also incorporated in TILDE. The only point where our algorithms C 0.5 and TILDE differ from the propositional TDIDT algorithm is in the computation of the tests to be placed in a node. To this aim, we employ a classical refinement operator under $\theta$-subsumption [12],see [2] for details.

Another point where $\mathrm{C} 0.5$ deviates from the classical TDIDT algorithm is in its heuristics. TDIDT algorithms typically use two heuristics: a splitting criterion to select the best test in a node, and a stopping criterion to decide whether a given node or cluster should be turned into a leaf or should be split further.

The splitting criterion used in $\mathrm{C} 0.5$ works as follows. For a given a cluster $C$, and a test $T$ that will result in two disjoint subclusters $C_{1}$ and $C_{2}$ of $C$, C 0.5 computes the distance $d\left(p\left(C_{1}\right), p\left(C_{2}\right)\right)$. The best test $T$ is then the one

\footnotetext{
${ }^{1}$ Using Plotkin's [12] notion of $\theta$-subsumption or the variants corresponding to structural matching $[1,13]$.
} 
that maximizes this distance. This reflects the principle that the inter-cluster distance should be as large as possible.

Stopping criteria for conceptual clustering are usually less clear. Typically, the stopping criterion depends on the number and size of clusters one wants to obtain. In C 0.5 , we consider right now only that each leaf of the tree should contain a minimal number of examples.

\section{Applications of Clustering in ILP}

We see a number of interesting applications of clustering in ILP. We will divide these into characterisation, classification and regression tasks. But first, we make an observation that will be important to all these tasks.

Once a decision tree has been induced, there can be two separate arguments for saying that an example belongs to a cluster. One is based on the tests in the tree. If an example is sorted into a specific node based on those tests, one has reason to say that the example really belongs to the cluster corresponding to that node. A second argument is based on the distance metric. Without performing any tests in the tree, one can still say that an example belongs to a cluster because it is close to the examples in that cluster. Both arguments are quite independent from one another (cf. also [10]).

This means that information can flow in two opposite directions. One can assign an example to a cluster based on the tree, and predict that its distance to the examples in that cluster will be small; or one can assign it to a cluster based on its distance to the examples in the cluster, and predict that it will satisfy the description of that cluster that the tree gives. While the first information flow has a flavour of classification, the second comes closer to characterisation (although the difference between the two is not always very clear-cut).

\subsection{Characterisation of clusters}

The fact that logical decision trees offer a first order description of clusters makes it possible to perform a kind of abduction. Based on the fact that an example belongs to a specific cluster (according to the distance metric that is used), one can predict that it fulfills the intensional description of that cluster.

\subsection{Classification}

It is quite clear that first order clustering can be used for classification; in fact, the original TILDE system is such a classification system. Following Langley's viewpoint, it is basically a clustering system where the "distance" metric is the class entropy within the clusters : lower class entropy within a cluster means that the examples in that cluster are more similar with respect to their classes.

Since TILDE needs information about the classes of its training examples, it is a supervised learning system. However, clustering can also be done in an unsupervised manner. The distance metric used to form clusters may or may not 
use information about the classes of the examples. Even if it does not use class information, clusters may be coherent with respect to the class of the examples in them. This will be illustrated in our experiments.

This principle leads to a classification technique that is very robust with respect to missing class information. Indeed, even if only a small percentage of the examples is labelled with a class, one could perform unsupervised clustering, and assign to each leaf in the concept hierarchy the majority class in that leaf. If the leaves are coherent with respect to classes, this method would yield high classification accuracy with a minimum of class information available. This is similar to Emde's method for learning from few classified examples, implemented in the COLA system [7].

\subsection{Regression}

The above shows that first order clustering can be used for characterisation of clusters in the data, as well as for classification. An application that has a flavour of both, is predicting numerical values. If clusters are coherent with respect to some numerical attribute of the examples, one can compute the average value for a cluster of examples and use this to predict the value of unseen examples. This is basically what Kramer's SRT system [10] does. SRT builds so-called structural regression trees: trees in which each leaf predicts a numerical value instead of a symbolic class and the tests in the nodes are conjunctions of literals.

\section{$5 \quad$ Experiments}

We have evaluated some of the ideas proposed in this paper using a preliminary implementation of C 0.5. Concerning the evaluation of a clustering system, there is the fundamental problem of how to evaluate the quality of a set of clusters as there is no clear and objective criterion to measure this. The situation is different in concept-learning, where the quality of the output of a system is measured in terms of accuracy. Therefore, as frequently done in conceptual clustering (cf. [9]), we will measure the quality of the obtained clusters using the accuracy with which these clusters can be used for prediction.

\section{$5.1 \quad$ Experimental setup}

Originally, mutagenicity was represented by a number on a logarithmic scale. For prediction purposes, a molecule is considered to be of class positive if this number is positive, and is of class negative otherwise. In all experiments we use euclidean distance metrics based on the numerical information about molecules, i.e. their logp, lumo and $\log m$ (which stands for log-mutagenicity, the number describing the activity of the molecule) values. Several distance metrics have been tried out. First of all, supervised learning (using logm for distance computation) was compared to unsupervised learning (using logp and lumo). This was done for three different hypothesis languages corresponding to the background 
knowledges BG1-BG3 as defined in [14]. BG1 contains only structural information (atoms and bonds), BG2 adds to this the charges of each individual atom, BG3 adds to BG2 the lumo and logp values of each molecule.

In order to evaluate the results of the clustering algorithm, we have computed accuracies as follows. For a whole cluster the $\log m$ value of the prototypical element is computed. If this is a positive number, then the whole cluster is predicted to be positive, otherwise negative. The observed class of an example is then compared with the class of its cluster. Classification accuracy on the regression-friendly set is based on tenfold crossvalidation, for the regressionunfriendly set a leave-one-out procedure was adopted.

\subsection{Classification Accuracy}

In a first experiment we compare supervised and unsupervised clustering, on both the regression-friendly and regression-unfriendly datasets. Table 1 shows the results of our experiments. The results for supervised learning can be compared with results obtained with other systems (Progol, TILDE), but for unsupervised learning this comparison is harder in the case of BG1 and BG2, since $\mathrm{C}$ 0.5 does use $\log p$ and lumo information during the induction process, even if the theories that are derived do not use them. For the regression-friendly data we have included results obtained with the TILDE system. The refinement operator and other parameters were exactly the same in C 0.5 as in TILDE, only the minimal coverage of clusters was higher (10 examples for the regression-friendly data, 4 for the regression-unfriendly data) because $\mathrm{C} 0.5$ does not have a good stopping criterion yet. We have not tried to find an optimal value for the minimal coverage, but consistently used 10 (or 4).

Table 1 shows that the results of both supervised and unsupervised learning are comparable with results for TILDE (with the exception of BG3 where a significantly lower score is obtained). Especially for unsupervised learning this is a surprise. It shows that lumo and logp are very closely related to the class; examples with similar lumo and logp values often have the same class. This explains in part why FOIL, on the Mutagenesis dataset with BG3, finds a theory simply consisting of a large number of intervals for logp and lumo (see [14]).

In order to check this out in more detail, we have additionally experimented (on BG2) with distances based on logp only, on lumo only, and on the three numerical values together. Results are shown in Table 2 (first row). There are no significant differences for the different distance functions.

The results on the regression-unfriendly set (Table 1) are much harder to explain. The expected behaviour (less accuracy for unsupervised than for supervised learning) occurs clearly in BG3, but not in BG1 and BG2. We do not have an explanation for this.

\subsection{Learning From Incomplete Examples}

Next to comparing different distance functions, Table 2 also shows what happens if numerical information is missing. The dataset was changed so that the lumo 
information was removed in approximately $50 \%$ of the models, chosen at random (every model has probability 0.5 of having this value removed), and the same was done independently for $\log p$ and $\log m$ values. This was repeated with removal of $75 \%$ or $90 \%$ of the information.

Table 2 shows that in the absence of a lot of information, classification accuracy slowly decreases. However, when using a distance measure that combines several numerical attributes, prototypes can be computed more accurately than if only one numerical attribute is used. Absence of information about one attribute can be compensated for by knowledge of another value. This causes the distance function using all three attributes (lumo, $\log p, \log m$ ) to be very robust w.r.t. missing information; predictive accuracy decreases more slowly.

These results suggest that even if more complicated distance functions do not yield higher accuracy when all information is present, they make learning from incomplete examples more feasible.

\begin{tabular}{|l|lll|lll|}
\hline & \multicolumn{3}{|c|}{ Regression friendly } & \multicolumn{3}{c|}{ Regression unfriendly } \\
& unsupervised supervised TILDE & unsupervised supervised TILDE \\
\hline BG1 & 0.73 & 0.74 & 0.75 & 0.76 & 0.76 & 0.83 \\
BG2 & 0.81 & 0.80 & 0.79 & 0.74 & 0.76 & 0.83 \\
BG3 & 0.79 & 0.78 & 0.85 & 0.71 & 0.86 & 0.79 \\
\hline
\end{tabular}

Table 1. Comparison of supervised and unsupervised clustering on both regressionfriendly and unfriendly data; results with TILDE included for reference.

\begin{tabular}{|c|ccccc|}
\hline $\begin{array}{c}\text { available } \\
\text { numerical data }\end{array}$ & \multicolumn{6}{|c|}{ lumo logp lumo+logp $\operatorname{logm}$ lumo+logp+logm } \\
\hline $100 \%$ & 0.79 & 0.79 & 0.81 & 0.80 & 0.81 \\
$50 \%$ & 0.77 & 0.79 & 0.81 & 0.78 & 0.79 \\
$25 \%$ & 0.73 & 0.72 & 0.73 & 0.72 & 0.77 \\
$10 \%$ & 0.65 & 0.65 & 0.67 & 0.67 & 0.74 \\
\hline
\end{tabular}

Table 2. Classification accuracies obtained on BG2 with several distance functions, and on several levels of missing information.

\section{Related Work}

Our work is related to KBG [1], which performs first order agglomerative clustering but employs a first order distance measure, to RIBL [8], a first order instance based learner, and to Kramer's SRT [10], which builds first order regression trees. Our work also exploits many of the ideas contained in Langley's book, as he makes the link between TDIDT and clustering. 


\section{Acknowledgements}

Luc De Raedt is supported by the Fund for Scientific Research, Flanders. Hendrik Blockeel is supported by the Flemish Institute for the Promotion of Scientific and Technological Research in the Industry (IWT). This work is also part of the European Community Esprit project no. 20237, ILP2. The authors wish to thank Luc Dehaspe, Wim Van Laer and Nico Jacobs for proofreading this text.

\section{References}

1. G. Bisson. Conceptual clustering in a first order logic representation. In Proceedings of the 10th European Conference on Artificial Intelligence, pages 458-462. John Wiley \& Sons, 1992.

2. H. Blockeel and L. De Raedt. Experiments with top-down induction of logical decision trees. Technical Report CW 247, Dept. of Computer Science, K.U.Leuven, January 1997. Also in Periodic Progress Report ESPRIT Project ILP2, January 1997.

3. L. De Raedt. Induction in logic. In R.S. Michalski and Wnek J., editors, Proceedings of the 3rd International Workshop on Multistrategy Learning, pages 29-38, 1996.

4. L. De Raedt and L. Dehaspe. Clausal discovery. Machine Learning, 26:99-146, 1997.

5. L. De Raedt and S. Džeroski. First order $j k$-clausal theories are PAC-learnable. Artificial Intelligence, 70:375-392, 1994.

6. L. De Raedt and W. Van Laer. Inductive constraint logic. In Proceedings of the 5th Workshop on Algorithmic Learning Theory, volume 997 of Lecture Notes in Artificial Intelligence. Springer-Verlag, 1995.

7. W. Emde. Inductive learning of characteristic concept descriptions. In S. Wrobel, editor, Proceedings of the 4 th International Workshop on Inductive Logic Programming, volume 237 of GMD-Studien, pages 51-70, Sankt Augustin, Germany, 1994. Gesellschaft für Mathematik und Datenverarbeitung MBH.

8. W. Emde and D. Wettschereck. Relational instance-based learning. In L. Saitta, editor, Proceedings of the 13th International Conference on Machine Learning, pages 122-130. Morgan Kaufmann, 1996.

9. D. H. Fisher. Knowledge acquisition via incremental conceptual clustering. $\mathrm{Ma}$ chine Learning, 2:139-172, 1987.

10. S. Kramer. Structural regression trees. In Proceedings of the 13th National Conference on Artificial Intelligence (AAAI-96), 1996.

11. P. Langley. Elements of Machine Learning. Morgan Kaufmann, 1996.

12. G. Plotkin. A note on inductive generalization. In Machine Intelligence, volume 5, pages 153-163. Edinburgh University Press, 1970.

13. L. De Raedt, P. Idestam-Almquist, and G. Sablon. Theta-subsumption for structural matching. In Proceedings of the 9th European Conference on Machine Learning, 1997.

14. A. Srinivasan, S.H. Muggleton, and R.D. King. Comparing the use of background knowledge by inductive logic programming systems. In L. De Raedt, editor, Proceedings of the 5th International Workshop on Inductive Logic Programming, 1995. 